\title{
Frequency and outcomes of endotracheal intubation in the pediatric emergency department
}

\author{
Ali Yurtseven ${ }^{1,2}$, Caner Turan ${ }^{1,2}$, Mehmet Arda Kılınçç,3, Eylem Ulaş Saz ${ }^{1,2}$ \\ Department of ${ }^{1}$ Pediatrics, ${ }^{2}$ Division of Emergency Medicine, ${ }^{3}$ Pediatric Intensive Care, Ege University School of Medicine, \\ Izmir, Turkey. E-mail: ali.yurtseven@ege.edu.tr \\ Received: 7th April 2017, Accepted: 19th May 2017
}

SUMMARY: Yurtseven A, Turan C, Kılınç MA, Saz EU. Frequency and outcomes of endotracheal intubation in the pediatric emergency department. Turk J Pediatr 2017; 59: 524-530.

Intubation is a core airway skill in Pediatric Emergency Medicine (PEM). The data on pediatric endotracheal intubation in the emergency department, especially in developing countries, is currently very limited. This study was designed to describe the frequency, clinical features and outcomes of pediatric intubation in a large children's hospital.

We performed a retrospective analysis of PEM medical records between January 2014 and December 2015 that involved any attempted intubations of children younger than 18 years. The medical records were reviewed to describe the intubation process, demographics, clinical features and outcomes.

A total of 110,000 patients visited our emergency department during the study period. Ninety-one of them $(1 / 1300)$ were intubated. The median age was 2 years, (F/M: 1) and 25 patients were younger than 12 months. Respiratory failure was the most common indication for intubation (42\%), followed by status epilepticus $(26 \%)$ and sepsis-shock (16\%). Mortality was associated with prolonged chest compression (more than 10 minute). A poor outcome was associated with sepsis-shock and cardiac diseases; however, better outcomes were associated with status epilepticus $(\mathrm{p}<0.001)$. The first attempt success (FAS) was achieved in 60/91 (66 \%) patients and the FAS rate was also higher in younger patients $(p=0.002)$. The discharge rate of all intubated patients in our study was sixty-four percent.

Pediatric residents performed the majority of intubations. A higher FAS rate was associated with younger patients. Favorable outcomes were directly related to duration of cardiopulmonary resuscitation and main diagnosis.

Key words: intubation, outcome, child, pediatric emergency department

Endotracheal intubation (ETI) one of the most common lifesaving interventions, is rarely performed in the emergency department (ED).1,2 There are, however, numerous disease processes and clinical situations that may necessitate immediate ETI; these include respiratory failure, cardiac arrest, shock, severe trauma and septic shock. This procedure is predominantly performed by healthcare professionals in prehospital settings and EDs. 3,4 Because intubations carry a significant risk of morbidity and mortality; especially in critically ill children, the appropriate ETI training of all healthcare professionals should be provided by all EDs. ${ }^{5}$
In Turkey most airway management procedures in both EDs and wards were traditionally done by anesthesiologists up until the early 1990's. As a medical specialty adult emergency medicine (EM) was only officially accepted in 1993. As a subspecialty within pediatrics, pediatric emergency medicine (PEM) was officially recognized 8 years later (2011). Following the establishment of both EM and PEM as specialties, emergency airway management, including rapid sequence intubation (RSI) by emergency physicians, has proven to be very effective in developed and developing countries. ${ }^{5-9}$ 
In our country, due to lack of trained pediatric emergency physicians, limited data on the magnitude of intubations conducted in pediatric emergency departments (PEDs) is currently available in the literature. This study aimed to determine frequency and outcomes of PED intubation in a tertiary hospital in Turkey. It also describes clinical features, indications of ETI and factors associated with first-attempt success (FAS).

\section{Material and Methods}

This retrospective, single-center cohort study was conducted at Ege University Hospital, Pediatric Emergency Department (PED) between January 2014 and December 2015. All patients intubated in the PED during the study period were included. FAS rate was calculated by comparing the number of patients on whom ETI was attempted with the times successful tracheal intubation was achieved on a first attempt at laryngoscopy. A tracheal intubation attempt was recognized as the single insertion of a laryngoscope blade into a subject's mouth, with the intent of inserting an endotracheal tube into the trachea. We defined successful intubation as the placement of an endotracheal tube distal to the glottis. This was judged to have occurred through a combination of clinical confirmations; particularly end-tidal $\mathrm{CO} 2$ measurement, and the patient's clinical course following intubation. Only patients on whom intubation was performed in the prehospital setting were excluded from our cohort.

Our PED is a tertiary-care teaching center; located in western of Turkey, it has 55,000 visits annually. The PED is served by 1 PEM attending staff, 2 PEM fellows, and 6 to 10 rotating residents per month. All residents who study in the PEM are supervised by attendings (pediatricians, PEM attending staff, PEM fellows).

Data was collected using a standard data collection form designed by the authors. This form contained information on the clinical and demographic characteristics of all study patients; this included age, sex, medical history, comorbidity, indications for intubation, factors associated with first attempt success, intubation process, drugs used during intubation, and outcome. All data was retrieved retrospectively from electronic medical records.
This study was approved by the local ethical committe.

\section{Statistical analysis}

All analyses were performed with SPSS for windows (ver. 19.0 SPSS Inc., IL, USA). Descriptive statistics were used to define the patient'demographic, intubation details, and outcome. Children were categorized into four age groups: infants (0-1year), toddlers (1-5 years), primary school children (5-10 years) and older children (10-18 years). As factors with potential to influence the FAS rate and the outcome, we focused patients' age, intubator's specialty, clinical diagnosis, the intubation method, comorbidity, shift time, prehospital medical transport and cardiopulmonary resuscitation (CPR). These were comparatively analysed using the Chi-square test and Fisher's exact test. A two-tailed probability value (p) of less than 0.05 was considered as significant.

\section{Results}

During the study period 110,000 patients visited our emergency department; of these $91(0.08 \%)$ were intubated. Fifty-one percent were male $(n=46)$ and the median age was 2 years. Regarding age; 25 were infants $(28 \%)$, 40 toddlers $(44 \%), 12(13 \%)$ primary school children and 14 (15\%) older children. Main underlying conditions for ETI were respiratory failure (42\%) and status epilepticus (26\%) (Table I).

Comorbidity was present in 61 patients $(67 \%)$; $24(39 \%)$ had central nervous system diseases, $14(23 \%)$ respiratory diseases, $10(16 \%)$ inborn errors of metabolism diseases, 7 (11\%) cardiovascular disease, 4 (7\%) malignancies, 1 (2\%) malnutrition and 1 (2\%) substance use.

Induction agents / sedatives were used in 64 cases $(70 \%)$ (midazolam/fentanyl in 56 cases $(88 \%)$ and ketamine in $8(12 \%))$. Rapid sequence intubation (RSI) was performed in 17 patients (19\%), and the most preferred neuromuscular blocking agent was rocuronium bromide used for 15 (17\%) patients (Table I).

Patients who presented with either sepsis-shock or heart failure were more likely to have a poor outcome. However better outcomes were associated with patients presenting with status epilepticus $(p<0.001)$ (Table II). 
Table I. Characteristics of Patients Who Received Endotracheal Intubation (N=91).

\begin{tabular}{lc} 
Characteristic & N $(\%)$ \\
\hline Age (years), median (range) & $2(0-17)$ \\
Sex & \\
Male & $46(51)$ \\
Female & $45(49)$ \\
Age group & \\
0-1 years & $25(28)$ \\
$1-5$ years & $40(44)$ \\
-10 years & $12(13)$ \\
10-18 years & $14(15)$ \\
Diagnosis & \\
Respiratory failure & $38(42)$ \\
Seizure & $24(26)$ \\
Sepsis/shock & $15(16)$ \\
Cardiac failure & $6(7)$ \\
Intoxication & $3(4)$ \\
Other & $5(6)$ \\
Cardiopulmonary arrest & \\
Yes & $31(34)$ \\
No & $60(66)$ \\
Comorbidities & \\
Yes & $61(67)$ \\
No & $30(33)$ \\
Sedative/paralytic use & \\
Midazolam & $56(62)$ \\
Fentanyl & $56(62)$ \\
Ketamine & $8(9)$ \\
Paralytic & $17(19)$ \\
\hline &
\end{tabular}

First attempt success was achieved in 60/91 (66 \%) patients, second attempt in $15 / 91$ (16.5\%), with 3 or more attempts being necessity in 16/91 (17.5\%). No patient required surgical interventions for airway. The majority intubations were performed by either pediatric residencies or PEM physician $56(61 \%)$ and $15(16 \%)$ respectively. While the FAS rate was associated with younger age $(p=0.002)$, no significant associations were found concerning the intubator's specialty or other variables (Table III).

In 31 patients (34\%) ETI was performed due to cardiopulmonary arrest with 17 of 31 remaining unrespondent to the CPR. Mortality was associated with prolonged chest compression (more than 10 minute). Three patients who developed cardiopulmonary arrest due to ventricular fibrillation required defibrillation.

Sixty-four percent of cases $(n=58)$ were successfully discharged home following admission to the pediatric intensive care unit. Of the patients receiving CPR (31), only 7 (\%23) were discharged following their PICU admission.

\section{Discussion}

This study was conducted due to a lack of data in the literature reporting the number of lifesaving interventions performed in the PED; such as resuscitation, ETI and advanced cardiac life support procedures. Our results have confirmed that critical illness requiring ETI occurs infrequently in the PED. This is consistent with previously published data which demonstrated the mean range of ETIs per 1,000 PED patients to be 0.8 to $3.3 .5,10,11$ This study is first to report the scope of ETI practice among PEDs in Turkey. The fact that our data is statistically similar to previous studies from other developed and developing countries, which also found low volumes of encounters with critical illness requiring 
resuscitation interventions in the PED, shows that our experience in a tertiary care PED is not unique. ${ }^{11,12}$

In this study, the majority of cohort were 1 to 5 years of age. Other studies, however, have showed most of the children are being younger than 1 year. ${ }^{1,13,14}$ There is a possible explanation for increased numbers of preschool children in our study. While our hospital has one of the most advanced pediatric intensive care units in available in Turkey, it has a comparatively smaller neonatal intensive care unit.

The most common clinical manifestations requiring ETI in this study was respiratory failure; this was followed by status epilepticus and sepsis-shock. Bano et al. ${ }^{9}$ reporting from a developing country, in conjuction with other studies from developed countries, showed similar results. That is, most pediatric ETIs are performed due to respiratory failure, cardiopulmonary arrest, seizure and trauma. ${ }^{10,15,16}$ Conversely however, Sagarin et al. ${ }^{7}$ found that the majority of the patients requiring ETI had presented with status epilepticus, followed by trauma. Due to the fact that adult EM in our institute cares for pediatric trauma patients, there were no trauma patients requiring ETI in our study.

Sixty-one patients (67\%) in the present study had comorbidity. Intubated patients did show better outcomes in the absence of comorbidity; however, the difference was not statistically significant. Similary, studies from other countries also indicated that most patients intubated in the EDs had comorbid conditions. No effect of comorbidity on outcomes, however, had been noted. ${ }^{9,16,17}$

Rapid sequence intubation is defined as the simultaneous administration of a sedative/ analgesic and neuromuscular blocking agent to render the patients rapidly unconscious and/or flaccid; in order to facilitate emergent ETI. ${ }^{18-20}$ In our study only $19 \%$ of intubations was performed using RSI. This RSI rate was lower than other studies. It can possibly be explained by the timing of most intubations.

Table II. Outcome of All Cases Who had Tracheal Intubation $(\mathrm{N}=91)$.

\begin{tabular}{lccc}
\hline Variables & $\begin{array}{c}\text { Discharged } \\
\mathrm{N}(\%)\end{array}$ & $\begin{array}{c}\text { Died } \\
\mathrm{N}(\%)\end{array}$ & P value \\
\hline Age group & $13(52)$ & $12(48)$ & 0.074 \\
$0-1$ years & $27(68)$ & $13(32)$ & \\
$1-5$ years & $11(92)$ & $1(8)$ & \\
5-10 years & $7(50)$ & $7(50)$ & 0.148 \\
10-18 years & $32(71)$ & $13(29)$ & \\
Sex & $26(56)$ & $20(44)$ & \\
Male & $24(63)$ & $14(37)$ & \\
Female & $22(92)$ & $2(8)$ & \\
Diagnosis & $6(40)$ & $9(60)$ & \\
Respiratory failure & $1(17)$ & $5(83)$ & \\
Seizure & $2(67)$ & $1(33)$ & \\
Sepsis/shock & $3(60)$ & $2(40)$ & \\
Cardiac failure & & & \\
Intoxication & $37(61)$ & $24(39)$ & \\
Other & $21(70)$ & $9(30)$ & \\
Comorbidity & & & \\
Yes & $35(60)$ & $23(40)$ & \\
No & $23(70)$ & $10(30)$ & \\
Shift hours & & & \\
$\quad$ Out of working hours & $19(54)$ & $16(46)$ & \\
Within working hours & $39(70)$ & $17(30)$ & \\
Prehospital medical transport & $7(23)$ & $24(77)$ & \\
Yes & $51(85)$ & $9(15)$ & \\
No & & & \\
Cardiopulmonary arrest & Yes & & \\
No & & & \\
\hline
\end{tabular}


Table III. Factors Associated with First Attempt Success (FAS) in Tracheal Intubation $(\mathrm{N}=91)$.

\begin{tabular}{lccc}
\hline Variables & FAS $^{\mathrm{n}} \mathrm{n}(\%)$ & Non-FAS $\mathrm{n}(\%)$ & P value \\
\hline Age group & & & \\
-1 years & $22(88)$ & $3(12)$ & 0.002 \\
1-5 years & $26(65)$ & $14(35)$ & \\
5-10 years & $8(67)$ & $4(33)$ & \\
10-18 years & $4(29)$ & $10(71)$ & 0.511 \\
Sex & $28(62)$ & $17(38)$ & \\
Male & $32(70)$ & $14(30)$ & \\
Female & & & 0.465 \\
Intubator & $12(80)$ & $3(20)$ & \\
PEM attending & $6(67)$ & $3(33)$ & \\
PEM fellow & $37(66)$ & $19(34)$ & \\
Pediatric resident & $4(50)$ & $4(50)$ & \\
Pediatric physician & $1(50)$ & $1(50)$ & \\
PICU attending & $0(0)$ & $1(100)$ & \\
Anesthetist & $14(82)$ & $3(18)$ & \\
Paralytic use & $46(62)$ & $28(38)$ & 0.251 \\
Yes & $36(62)$ & $22(38)$ & \\
No & $24(73)$ & $9(27)$ & \\
Shift hours & $20(65)$ & $11(35)$ & \\
Out of working hours & $40(67)$ & $20(33)$ & \\
Within working hours & & \\
Cardiopulmonary arrest & & \\
Yes & &
\end{tabular}

PEM: pediatric emergency medicine, PICU: pediatric intensive care unit

That is, they were performed outside of general working hours. ${ }^{10,20}$ Because the majority of intubators were pediatric residents and they have limited experience in airway mangament procedures, RSI was generally not performed.

Several investigators have tried to correlate 'the ETI of children' with 'the subsequent outcomes'. In one prospective observational study, Eich et al. ${ }^{21}$ focussed on prehospital ETIs performed by anaesthesia-trained emergency physicians, and found that the survival and neurological outcome was better in older children than infants. Another study conducted in the prehospital setting compared 'outcomes of pediatric patients treated with bag-valvemask ventilation only' with 'treated ETIs." ${ }^{22}$ The survival rate to hospital discharge in both group was low and there was no notable benifet to either. In the PED setting; a poor outcome was generally associated with: patients younger than 2 years old, arrival by prehospital medical transport and respiratory insufficiency on admission. ${ }^{14}$ Similarly, our results have also demonstrated that children who received ETI due to sepsis-shock and cardiac diseases are more likely to have a poor outcome. For patients with status epilepticus, however, we saw a better outcome. This is consistent with the literature.

First-attempt success rates ranging between 57\%- $83 \%$ have also been reported. ${ }^{23-26}$ Our result, with a FAS rate of $66 \%$, was consitent with this range. A correlation showing ETI success to be more likely as age increases also exists in the literature. In contrast to this, our results indicate that increased age is associated with a decrease in FAS rate. We believe that this difference may be explained by the timing of most intubations (outside of general working hours) and the training level of intubators in our study. Because pediatric residents generally work in out of hours shifts, they are the doctors performing most ETI. Intrestigly, these resident doctors, are on sequential rotation, coming directly from neonatal intensive care prior to pediatric intensive care and PEM. As one might expect, they are more familiar with anatomy of infant airways than older children, and hence more likely to attempt a complex procedure.

In several studies, it has been reported that intubation by emergency physicians is associated with a significantly higher chance of FAS ${ }^{23-25}$. A significantly higher FAS rate 
was also achieved when RSI was used; as compared to other methods ${ }^{19,20}$. No statistical difference in our study was noted regarding intubation method and intubators. However, we did observe that the FAS rates were better in patients intubated by a PEM attending. This can be explained by a low RSI rate.

In this study 31 patients (34\%) were intubated for cardiopulmonary arrest and the overall survival rate was $64 \%$. Similarly Eich et al. ${ }^{21}$ also showed an overall survival rate of $66 \%$, with $33 \%$ of their patients receiving chest compressions.

There were several limiting factors associated with this study. Firstly this was a retrospective study, hence, all complications were not fully documented and it was not possible to assimilate properly whether the physicians had followed all steps of ETI in accordance with the protocols. Secondly it was conducted in a single center and therefore, its results cannot be generalized to other settings.

In this study, 91 pediatric endotracheal intubations were performed within a 2 year period in the pediatric emergency department. The primary clinical manifestation for ETI was respiratory failure. A poor outcome was associated with sepsis-shock and cardiac failure, while better outcomes were associated with status epilepticus. First-attempt success rate found to be more likely as age decreased. During the ETI, drugs for sedation/analgesia are often used but neuromuscular-blocking agents were underused.

\section{REFERENCES}

1. Easley RB, Segeleon JE, Haun SE, Tobias JD. Prospective study of airway management of children requiring endotracheal intubation before admission to a pediatric intensive care unit. Crit Care Med 2000; 28: 20582063.

2. Carroll CL, Spinella PC, Corsi JM, Stoltz P, Zucker AR. Emergent endotracheal intubations in children: be careful if it's late when you intubate. Pediatr Crit Care Med 2010; 11: 343-348.

3. Ehrlich PF, Seidman PS, Atallah O, Haque A, Helmkamp J. Endotracheal intubations in rural pediatric trauma patients. J Pediatr Surg. 2004; 39: 1376-1380.

4. Sakles JC, Chiu S, Mosier J, Walker C, Stolz U. The importance of first pass success when performing orotracheal intubation in the emergency department. Acad Emerg Med 2013; 20: 71-78.
5. Losek JD, Olson LR, Dobson JV, Glaeser PW. Tracheal intubation practice and maintaining skill competency: Survey of pediatric emergency department medical directors Pediatr Emerg Care 2008; 24: 294-299.

6. Mittiga MR, Geis GL, Kerrey BT, Rinderknecht AS. The spectrum and frequency of critical procedures performed in a pediatric emergency department: Implications of a provider-level view. Ann Emerg Med 2013; 61: 263270.

7. Sagarin MJ, Chiang V, Sakles JC, et al. Rapid sequence intubation for pediatric emergency airway management. Pediatr Emerg Care 2002; 18: 417-423.

8. Kendirli T, Caltik A, Duman M, et al. Effect of pediatric advanced life support course on pediatric residents' intubation success. Pediatr Int 2011; 53: 94-99.

9. Bano S, Akhtar S, Zia N, Khan UR, Haq AU. Pediatric endotracheal intubations for airway management in the emergency department. Pediatr Emerg Care 2012; 28: 1129-1131.

10. Guilfoyle FJ, Milner R, Kissoon N. Resuscitation interventions in a tertiary level pediatric emergency department: implications for maintenance of skills. CJEM 2011; 13: 90-95.

11. Long E, Sabato S, Babl FE. Endotracheal intubation in the pediatric emergency department. Paediatr Anaesth 2014; 24: 1204-1211.

12. Chen EH, Shofer FS, Baren JM. Emergency medicine resident rotation in pediatric emergency medicine: What kind of experience are we providing? Acad Emerg Med 2004; 11: 771-773.

13. Lee JH, Turner DA, Kamat P, et al. The number of tracheal intubation attempts matters! A prospective multi-institutional pediatric observational study. BMC Pediatr 2016; 16: 58.

14. Claudet I, Bounes V, Fédérici S, et al. Epidemiology of admissions in a pediatric resuscitation room. Pediatr Emerg Care 2009; 25: 312-316.

15. Nishisaki A, Turner DA, Brown CA, Walls RM, Nadkarni VM. A National Emergency Airway Registry for children: Landscape of tracheal intubation in 15 PICUs. Crit Care Med 2013; 41: 874-885.

16. Sukys GA, Schvartsman C, Reis AG. Evaluation of rapid sequence intubation in the pediatric emergency department. J Pediatr (Rio J) 2011; 87: 343-349.

17. Miller KA, Kimia A, Monuteaux MC, Nagler J. Factors associated with misplaced endotracheal tubes during intubation in pediatric patients. J Emerg Med 2016; 51: 9-18.

18. Zuckerbraun NS, Pitetti RD, Herr SM, et al. Use of etomidate as an induction agent for rapid sequence intubation in a pediatric emergency department. Acad Emerg Med 2006; 13: 602-609.

19. Sagarin MJ, Barton ED, Chng YM, Walls RM. Airway management by US and Canadian emergency medicine residents: A multicenter analysis of more than 6,000 endotracheal intubation attempts. Ann Emerg Med 2005; 46: 328-336. 
20. Kerrey BT, Rinderknecht AS, Geis GL, Nigrovic LE, Mittiga MR. Rapid sequence intubation for pediatric emergency patients: Higher frequency of failed attempts and adverse effects found by video review. Ann Emerg Med 2012; 60: 251-259.

21. Eich C, Roessler M, Nemeth M, et al. Characteristics and outcome of prehospital paediatric tracheal intubation attended by anaesthesia-trained emergency physicians. Resuscitation 2009; 80: 1371-1377.

22. Gausche M, Lewis RJ, Stratton SJ, et al. Effect of outof-hospital pediatric endotracheal intubation on survival and neurological outcome: A controlled clinical trial. JAMA 2000; 283: 783-790.
23. Goto T, Gibo K, Hagiwara Y, et al. Factors associated with first pass success in pediatric intubation in the emergency department. West J Emerg Med 2016; 17: 129-134.

24. Choi HJ, Je SM, Kim JH, Kim E. The factors associated with successful pediatric endotracheal intubation on the first attempt in emergency departments: A 13-emergency-department registry study. Resuscitation 2012; 83: 1363-1368.

25. Sanders RC, Giuliano JS, Sullivan JE, et al. Level of trainee and tracheal intubation outcomes. Pediatrics 2013; 131: 821-828.

26. Caltık A, Kendirli T, İnce E. Endotracheal intubation experiences in pediatric intensive care patients. Ankara Üniversitesi Tıp Fakültesi Mecmuası 2006; 59: 93-97. 